\title{
Alternative Combined Retrograde-Antegrade Use of a Re-entry Device in the Revascularization of a Surgically Ligated Superficial Femoral Artery Occlusion
}

\author{
Roberto Gandini ${ }^{1} \quad$ Armando Raso $^{1} \quad$ Arezia Di Martino $^{1} \quad$ Fabio Salimei ${ }^{1} \quad$ Daniele Morosetti $^{1}$ \\ ${ }^{1}$ Department of Diagnostic Imaging and Interventional Radiology, \\ Address for correspondence Armando Raso, MD, Department \\ University of Tor Vergata, Rome, Italy \\ of Diagnostic Imaging and Interventional Radiology, University \\ Hospital “Tor Vergata," Viale Oxford, 81, 00133 Rome, Italy \\ (e-mail: armando.raso87@gmail.com).
}

J Clin Interv Radiol ISVIR:2021;5:62-65

\begin{abstract}
We report a case of a diabetic patient with critical limb ischemia, who previously underwent thromboendarterectomy at the right lower extremity, resulting in surgical ligation at the proximal third of the right superficial femoral artery (SFA). Twenty months later, the patient developed foot ulcers; endovascular treatment was therefore performed. After obtaining a retrograde right SFA subintimal access, directly puncturing the occluded segment of the artery, a re-entry intravascular ultrasound-guided

Keywords

- surgical ligation

- revascularization

- re-entry catheter was used to gain proximal re-entry. Then, the same device was used again, in antegrade fashion, to obtain re-entry into the patent popliteal artery. A covered stent was deployed in the site of the surgical closure. In the following 3 months, foot ulcers healed.
\end{abstract}

\section{Introduction}

Critical limb ischemia is the "end stage" of the peripheral arterial disease, a clinical condition representing an impending threat of amputation for many diabetic patients. Their hope resides in revascularization procedures, especially endovascular ones, thanks to the technological and technical advances made in the recent years. ${ }^{1}$

Still, these patients' arterial chronic total occlusions hinder technical success. In these cases, subintimal approach and an expert use of re-entry devices may help interventionists to accomplish arterial revascularization. ${ }^{2}$

\section{Case Report}

A 73-year-old male diabetic patient presented at our department because of the appearance of nonhealing ulcers at the first finger of the right foot, 20 months after right common femoral artery (CFA) thromboendarterectomy, resulting in surgical transection and ligation of the first segment of the superficial femoral artery (SFA). The transcutaneous oxygen pressure $\left(\mathrm{TCPO}_{2}\right)$ at ulcer site was $10 \mathrm{~mm} \mathrm{Hg}$.

The following computed tomography (CT) angiography examination showed regular patency of the right CFA, SFA occlusion at its proximal third, which was the site of extensive calcifications, and patency restoration at the Hunter channel (occlusion length: $34 \mathrm{~cm}$ ). Popliteal artery, tibioperoneal trunk, posterior tibial, and plantar arteries were patent, while the proximal third of the anterior tibial and peroneal arteries resulted in occlusion. The patient had severe comorbidities, such as obesity and chronic obstructive pulmonary disease with chronic respiratory insufficiency.

The procedure was performed after a 7-day double-antiplatelet therapy (acetylsalicylic acid $100 \mathrm{mg}$ and clopidogrel $75 \mathrm{mg} / \mathrm{die}$ ). Before the procedure, the patient signed an informed written consent.

Digital subtracted angiography, after antegrade right CFA access, confirmed CT findings ( - Fig. 1A, B). Soon after sheath
DOI https://doi.org/ 10.1055/s-0041-1723097 ISSN 2457-0214. (c) 2021. Indian Society of Vascular and Interventional Radiology. This is an open access article published by Thieme under the terms of the Creative Commons Attribution-NonDerivative-NonCommercial-License, permitting copying and reproduction so long as the original work is given appropriate credit. Contents may not be used for commercial purposes, or adapted, remixed, transformed or built upon. (https://creativecommons.org/licenses/by-nc-nd/4.0/).

Thieme Medical and Scientific Publishers Pvt. Ltd. A-12, 2nd Floor, Sector 2, Noida-201301 UP, India 
insertion, low-weight heparin was systemically administered $(2,500 \mathrm{IU} / \mathrm{h})$. Multiple attempts were made to cross the occlusion using a straight-tip 0.035" stiff guidewire (Radifocus Terumo), with the support of a $4 \mathrm{~F}$ vertebral catheter (Cordis), but result was unsuccessful, due to unengageable occlusion of the proximal SFA.

Using the arterial calcifications as a landmark, the occluded SFA was punctured under fluoroscopic guidance with an 18G Seldinger needle at its proximal third, 3 to $4 \mathrm{~cm}$ below the occlusion ( - Fig. 1C). Then, a 0.035 " stiff guidewire and a 6Fr long introducer sheath (Radifocus Terumo) were retrogradely advanced in the subintimal space until reaching the SFA origin ( - Fig. 1D), to safeguard the Pioneer device, and the wire was switched to a 0.014 " one (Pilot 200) to allow its delivery.
Thus, the 6F Pioneer Plus catheter (Volcano Therapeutics) was retrogradely advanced over the Pilot wire and its position and rotation were adjusted under combined intravascular ultrasound and fluoroscopic guidance. The true arterial lumen at the level of CFA bifurcation was successfully punctured ( - Fig. 1E).

The subintimal channel was predilated using a $4 \times 120 \mathrm{~mm}$ balloon catheter (Amphirion Deep, Invatec) ( - Fig. 2A), except the re-entry site, and the $6 \mathrm{~F}$ Pioneer was antegradely advanced until reaching the popliteal artery ( - Fig. 2B). Once gained both intraluminal connections, the whole subintimal channel was dilated, from distal to proximal, using a $5 \times 150 \mathrm{~mm}$ mono-rail balloon catheter (Senri, Terumo) (-Fig. 2C). A $6 \times 20 \mathrm{~mm}$ balloon-expandable covered stent (Begraft, Bentley) was deployed across the surgical ligation
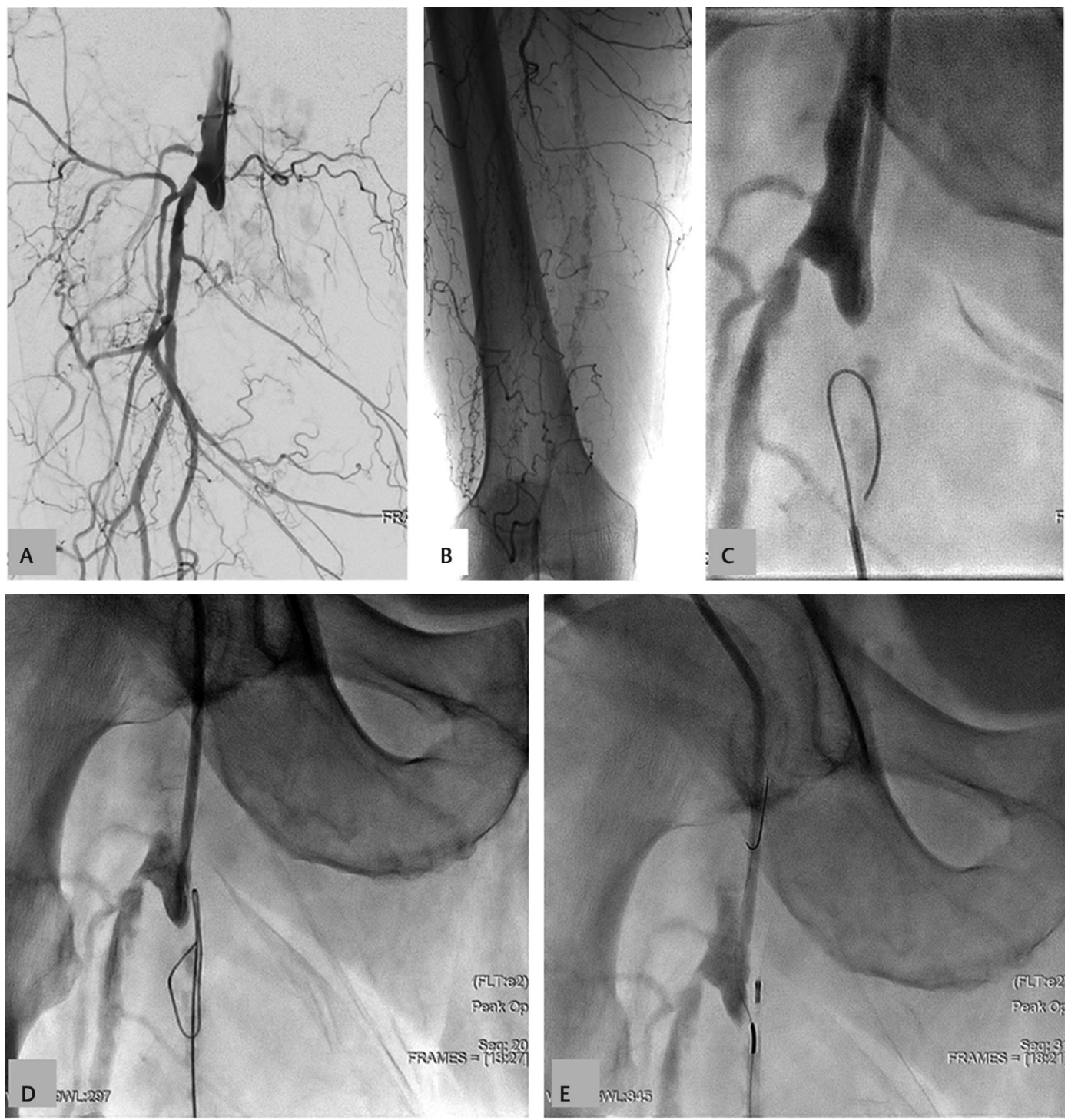

Fig. 1 (A, B) First angiograms show occlusion of the superficial femoral artery (SFA) with rehabilitation at the level of the popliteal artery. The whole artery is site of extensive calcifications. (C, D) The SFA is retrogradely punctured at its proximal third, in the occluded segment, using the calcifications as a marker. A looped wire is pushed in the subintimal space along the occluded vessel. (E) After exchanging the 0.035 " guidewire with a 0.014 " one, the Pioneer device is delivered in the subintimal space and adjusted to reach the minimal distance between the needle tip and the true lumen. Then, intraluminal re-entry is accomplished under intravascular ultrasound guidance and the 0.014 " wire snared from the tip of the antegrade sheath. 

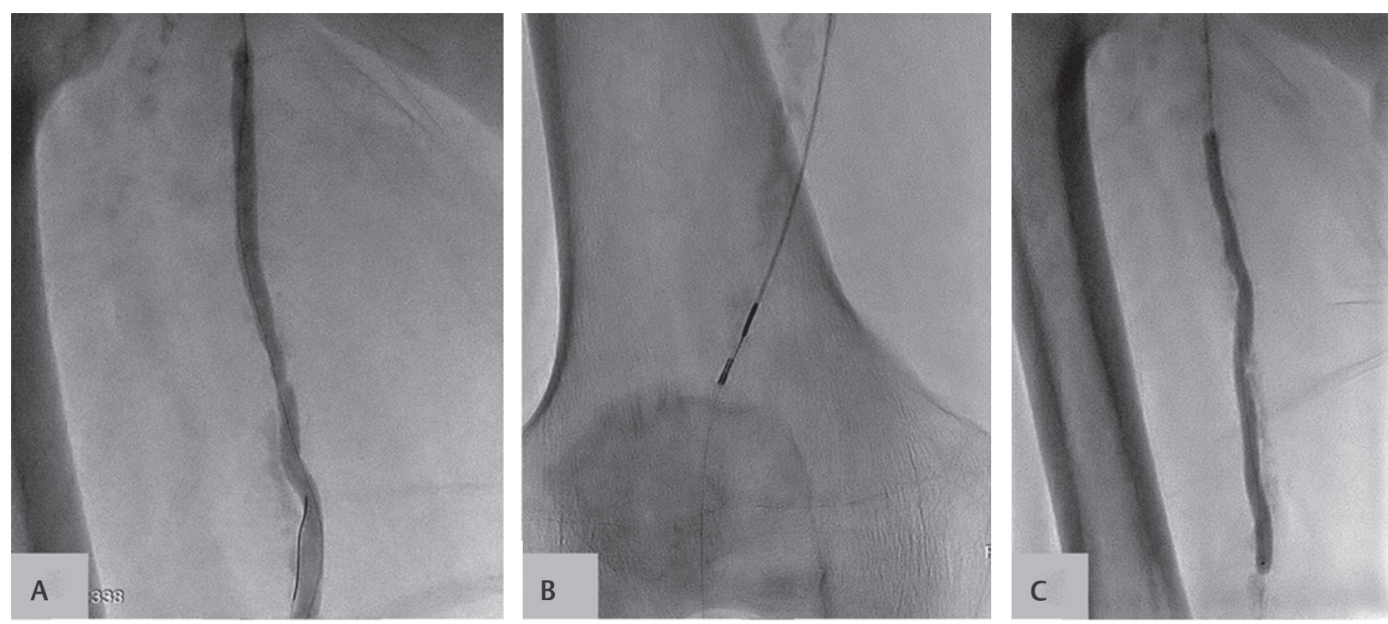

Fig. 2 (A) Subintimal space is predilated and the wire is driven to the level of the popliteal artery. (B) The Pioneer catheter is used again, this time in antegrade fashion, to gain intraluminal re-entry at the level of the distal patent artery. (C) The occluded segment is dilated with a $4 \times$ $120 \mathrm{~mm}$ balloon-catheter from distal to proximal.

site. Final angiography showed regular stent and SFA patency and caliber ( - Fig. 3A, B).

At the 3-month follow-up visit, the patient presented a healed ulcer and increased $\mathrm{TCPO}_{2}(58 \mathrm{~mm} \mathrm{Hg})$. Arterial flow was further detectable through Doppler imaging.

\section{Discussion}

Several reviews show the advantages of using re-entry devices when treating complex iliac or femoral chronic total occlusions, in terms of enhanced technical success and safety of revascularization procedures, decreased dissection risk by precise re-entry, and reduced operative time and fluoroscopic exposure. ${ }^{3,4}$
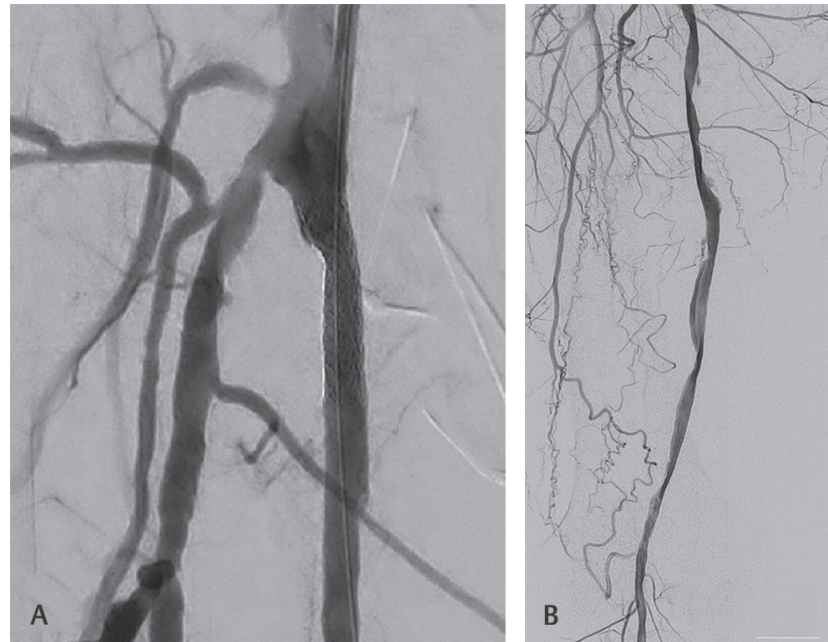

Fig. 3 (A) A balloon-expandable covered stent is deployed in the proximal superficial femoral artery (SFA), across the site of the surgical ligation. (B) Control angiography shows SFA and stent patency and restored SFA caliber.
In this case, we opted for puncturing the SFA in the occluded segment. The arterial calcifications drove the puncture, giving access to the subintimal plane. The proximal third of the SFA was chosen to minimize the pathway to the re-entry point and to improve the system pushability in the subintimal space. Moreover, we introduced the Pioneer catheter inside the sheath previously placed in the subintimal space, to facilitate its delivery in the occluded arterial segment.

We performed the retrograde SFA puncture under both intravascular ultrasound (IVUS) and fluoroscopic guidance to choose the minimal distance between the subintimal space and the true lumen. The aim obviously was to make an effective intraluminal puncture and, as a result, to reduce the risk of arterial rupture by the following angioplasty.

The first subintimal percutaneous transluminal angioplasty was performed to create an appropriate channel for advancing the Pioneer catheter, but the proximal re-entry site was not dilated at first, because without a distal re-entry, it would expose the artery to the risk of rupture. Once obtained distal re-entry, the subintimal channel was dilated from distal to proximal except for the proximal re-entry site.

Considering the high risk of bleeding, after this maneuver, we deployed a short balloon-expandable covered stent across the proximal, previously ligated, re-entry site, thus creating a sort of endovascular bypass over a suspended arterial segment and thus completing the angioplasty of the occluded segment.

\section{Conclusion}

The revascularization of an occluded artery can be efficiently accomplished by a combined retrograde and antegrade use of the Pioneer IVUS-guided re-entry device, even in case of a surgically ligated artery. 


\section{Conflict of Interest}

None.

\section{References}

1 Norgren L, Hiatt WR, Dormandy JA, Nehler MR, Harris KA, Fowkes FG; TASC II Working Group. Inter-Society Consensus for the Management of Peripheral Arterial Disease (TASC II). J Vasc Surg 2007;45(Suppl S) :S5-S67

2 Gandini R, Fabiano S, Spano S, et al. Randomized control study of the outback LTD reentry catheter versus manual reentry for the treatment of chronic total occlusions in the superficial femoral artery. Catheter Cardiovasc Interv 2013;82(3):485-492
3 Jacobs DL, Motaganahalli RL, Cox DE, Wittgen CM, Peterson GJ. True lumen re-entry devices facilitate subintimal angioplasty and stenting of total chronic occlusions: Initial report. J Vasc Surg 2006;43(6):1291-1296

4 Krishnamurthy VN, Eliason JL, Henke PK, Rectenwald JE. Intravascular ultrasound-guided true lumen reentry device for recanalization of unilateral chronic total occlusion of iliac arteries: technique and follow-up. Ann Vasc Surg 2010;24(4):487-497 